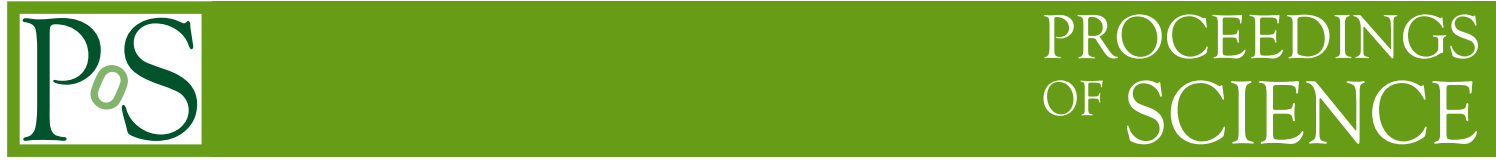

\title{
Advances and applications of lattice supersymmetry
}

Joel Giedt*

Fine Theor. Phys. Inst., Univ. of Minnesota, 116 Church St. S.E., Minneapolis, MN 55455 USA

E-mail: giedt@physics.umn.edu

I review motivations for the study of supersymmetric field theories by lattice techniques. In particular, some of the more interesting potential applications are described. These are models of quantum gravity, that rely on the AdS/CFT correspondence. The dual gauge theory is an ideal place for lattice studies to make a contribution. I also survey some of the recent advances in lattice formulations of supersymmetric theories.

XXIV International Symposium on Lattice Field Theory

July 23-28 2006

Tucson Arizona, US

\footnotetext{
*Speaker.
} 


\section{Introduction}

Several motivations exist for efforts to formulate supersymmetric field theories on a lattice. As is well known, it is difficult to formulate these theories in a way that avoids fine-tuning of counterterms. Nevertheless, there have been many promising formulations developed of late. ${ }^{1}$ But, given a "good" formulation, to what use should or would we put it? To answer this question, let us look to the motivations for putting supersymmetric theories on the lattice.

\section{To provide a definition of the field theory}

\subsection{Some general remarks}

Here, one wants to say in a precise way what is meant by the rather formal expression

$$
Z[S]=\int \exp (-S)
$$

the Euclidean functional integral, or partition function for the system. At a formal level we know that it is described by imposing an ultraviolet (UV) cutoff length scale $a$, and defining the integral for each $a$ :

$$
Z[S ; a]=\int_{\mathscr{D}(a)}[d \mu(\phi ; a)] \exp (-S[\phi ; a])
$$

This leads to an $a \rightarrow 0$ limiting sequence that defines the physical theory. In the lattice formulation, $a$ is of course the lattice spacing, and $a \rightarrow 0$ is the continuum limit.

Due to universality, there is no unique definition of $Z[S ; a]$. That is, two definitions that differ by irrelevant operators can be made to agree in their continuum limit. But among various proposals for $Z[S ; a]$, one wants a classification of the extent to which local counterterms (CTs) need to be adjusted to obtain the correct continuum limit. The best definitions of $Z[S ; a]$ possess the continuum symmetries (e.g., the exact gauge symmetry of Wilson's action), or have lattice symmetries that guarantee the continuum symmetries are recovered as $a \rightarrow 0$ (e.g., the lattice chiral symmetry of Ginsparg-Wilson fermions). In either case, the continuum symmetry results without any adjustment of CTs. In some cases, the lattice theory explicitly breaks continuum symmetries, and the symmetries are only obtained through a fine-tuning of CTs. It may even be that no local CTs exist that will do the job, in which case we have an anomaly. We should study the question of CTs both perturbatively and nonperturbatively. Our favorite answer of course would be that no symmetry-restoring CTs are required. ${ }^{2}$

\subsection{Examples without CTs}

I will now enumerate several examples where no CTs are required. The point here is to familiarize the reader with the many successes, or claimed successes, that have been achieved in this regard. Some of these are quite recent.

\footnotetext{
${ }^{1}$ For recent reviews, see [1-5].

${ }^{2}$ Of course, CTs associated with renormalization are required unless the theory is finite. But these are CTs allowed by the continuum symmetry and will not concern us here.
} 


\begin{tabular}{ccc}
\hline$N$ & $m_{B}$ & $m_{F}$ \\
\hline 16 & $10.60(3)$ & $10.64(5)$ \\
32 & $12.80(2)$ & $12.91(4)$ \\
64 & $14.47(2)$ & $14.52(2)$ \\
128 & $15.63(4)$ & $15.63(4)$ \\
256 & $16.19(3)$ & $16.28(4)$ \\
\hline
\end{tabular}

Table 1: Spectrum degeneracy in Q-exact supersymmetric quantum mechanics. These results are from [6]. Note that with $L=N a=$ fixed, increasing $N$ corresponds to the continuum limit. The $N \rightarrow \infty$ extrapolation matches the (numerical) solution of the continuum Schrödinger equation.

\subsubsection{Q-exact supersymmetric quantum mechanics}

In this case, one has a lattice version of a supercharge $Q$ that is nilpotent; i.e. $Q^{2}=0$. The lattice action is describable as a $Q$ acting on something, " $X$ ": $S=Q X$, and is trivially invariant: $Q S=Q^{2} X=0$.

There is convincing Monte Carlo evidence that the lattice theory in this case has the correct continuum limit without fine-tuning of CTs. This evidence consists of Bose-Fermi spectrum degeneracy and supersymmetry Ward identities [6]. For example, in Table 1 one sees that the mass gap for fermionic states is degenerate with that for bosonic states, even at finite lattice spacing.

An all orders perturbative proof of the absence of CTs in this theory was given in [7]. Moreover, a nonperturbative proof of the absence of CTs was also given in [7], using transfer matrix methods. In either calculation, it is apparent that the Q-exact nature of the action leads to cancellations that avoid the appearance of CTs. These cancellations are associated with the exact supersymmetry Ward identities that follows from $Q S=0$.

\subsubsection{Q-exact 2d $\mathscr{N}=2$ Wess-Zumino (a.k.a. $\mathscr{N}=2$ Landau-Ginsburg) model}

Here, the model is the $4 \mathrm{~d} \rightarrow 2 \mathrm{~d}$ dimensional reduction of the Wess-Zumino model with a cubic superpotential [8]. The Q-exact lattice formulation of this model was given long ago $[9,10]$. An all orders perturbative proof of the absence of CTs was given in [7]. That proof is similar to the one for the $Q, Q^{\dagger}$-preserving spatial lattice [9]. In both cases, Q-exactness leads to supersymmetry Ward identities, which cause the cancellations that avoid CTs. Fig. 1 shows all the diagrams that must be studied (the lattice theory is super-renormalizable). The parts that would potentially differ from the continuum perturbation series, according to Reisz's theorem [11], all cancel.

On the nonperturbative front, there is various numerical evidence (spectrum degeneracy, Ward identities, R-symmetry) that the supersymmetric limit is obtained without CTs [12-15].

\subsection{3 $\mathscr{N}=1$ 4d super-YM with Ginsparg-Wilson fermions}

From the work of [16], we know that $\mathscr{N}=14 \mathrm{~d}$ super-YM (SYM) with Ginsparg-Wilson fermions requires no supersymmetry-restoring CTs. This is because the chiral limit and the supersymmetric limit in this model are equivalent: the only gauge invariant relevant operator that could break supersymmetry is a gaugino mass. The application of overlap fermions [17-19] to 


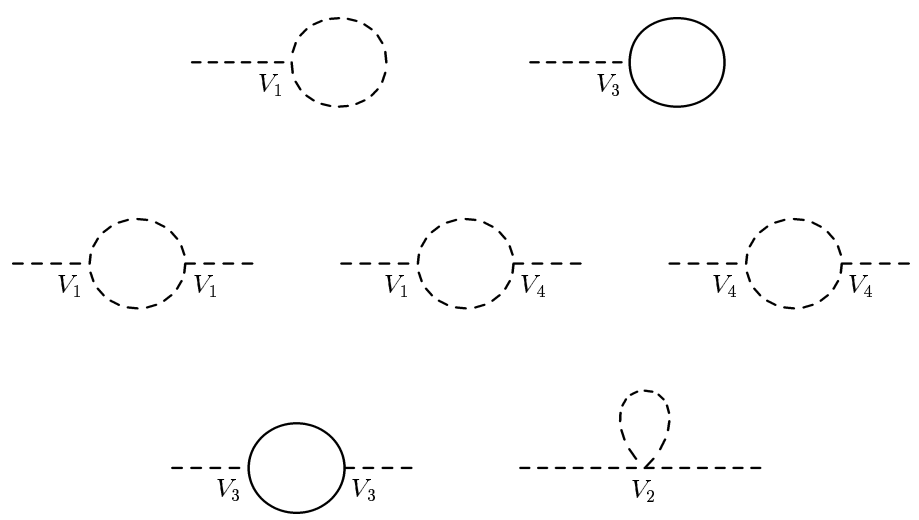

Figure 1: The set of all 1PI diagrams with UV degree of divergence $D_{U V} \geq 0$ in the Q-exact lattice $2 \mathrm{~d}$ $\mathscr{N}=2$ Wess-Zumino model. The notation for the vertices, which are modified on the lattice, is explained in [7].

this model was sketched in [20,21]. But leading order simulation studies, such as glueball spectra, have yet to be attempted. The application of domain wall Dirac fermions [22] to this model was proposed [21,23], sketched [24] and briefly studied [25]. However, it deserves another push: much more could be accomplished with the computational resources that are now available. ${ }^{3}$ Infrared (IR) effective theories for this model have been proposed by continuum theorists, based on various arguments [26-32]. In particular, the predicted IR spectrum of states in the presence of a small gluino mass has a pattern of splitting [28] that would be very interesting to test in a lattice formulation that is sensitive to topology, such as gap domain wall fermions [33].

\subsubsection{Deconstruction models}

Deconstruction models (a.k.a. quiver lattice, orbifold matrix model) offer another route that avoids some or all CTs. ${ }^{4}$ These have been written down for various extended SYM and superQCD models in 2d, 3d and 4d [36-41]. It is easily shown, on symmetry and dimensional grounds, that the $2 \mathrm{~d}$ SYM examples require no supersymmetry-restoring CTs in perturbation theory. In the case of $2 \mathrm{~d}$ super-QCD, renormalization has yet to be explored. For the $d>2$ examples, it is less clear what really happens; explicit calculations will be required. However, if the Euclideanized ddimensional Poincaré group is recovered, an interesting supersymmetric theory is obtained either way, since there is at least some supersymmetry present, even if not the full supersymmetry of the target theory. ${ }^{5}$ A review of the deconstruction models was recently given in [1]. Perturbative

\footnotetext{
${ }^{3}$ I thank Pavlos Vranas for extensive conversations on what could be achieved presently.

${ }^{4}$ These models are inspired by earlier, phenomenological works on dimensional deconstruction [34, 35].

${ }^{5}$ Thanks are due David Kaplan for emphasizing this point during conversations.
} 
aspects of one of the models was studied in [42]. In [43-45], the phase of the fermion determinant was found to be problematic in two of the models.

\subsubsection{D'Adda et al. SYM}

Extended SYM in $2 \mathrm{~d}$ and $4 \mathrm{~d}$ has been formulated in a very interesting way by D'Adda et al. [46,47]. It is based on a modified Leibnitz rule for the supersymmetry variations $s_{A}$ of products of fields:

$$
\begin{gathered}
s_{A}\left[\Phi\left(x_{1}\right) P\left(x_{2}, \ldots\right)\right]=s_{A}\left[\Phi\left(x_{1}\right)\right] P\left(x_{2}, \ldots\right) \\
+(-)^{F(\Phi)} \Phi\left(x_{1}+a_{A}\right) s_{A}\left[P_{2}\left(x_{2}, \ldots\right)\right] .
\end{gathered}
$$

Note that the site index $x_{1}$ of $\Phi$ has shifted as $s_{A}$ is pushed past it. It is in this sense that the formulation is "slightly noncommutative."

For the $2 \mathrm{~d}(2,2)$-supersymmetric case, the action can be written in four equivalent forms:

$$
\begin{aligned}
S & =\sum_{x} \operatorname{Tr} s \tilde{s} s_{1} s_{2} \Psi_{x, x}=-\sum_{x} \operatorname{Tr} \tilde{s} s s_{1} s_{2} \Psi_{x, x} \\
& =\sum_{x} \operatorname{Tr} s_{1} s_{2} s \tilde{s} \Psi_{x, x}=-\sum_{x} \operatorname{Tr} s_{2} s_{1} s \tilde{s} \Psi_{x, x}
\end{aligned}
$$

where $\Psi_{x, x}$ is a composite operator whose exact form we will not need here. Due to the nilpotentcy of the supersymmetry operators $s_{A}$,

$$
s^{2}=\tilde{s}^{2}=s_{1}^{2}=s_{2}^{2}=0,
$$

the action is invariant under the modified $(2,2)$ supersymmetry. The renormalization of the model needs more study. In particular, does the slight noncommutativity matter?

Bruckmann and de Kok have studied the noncommutativity approach in supersymmetric quantum mechanics [48]. They find that the existence of modified supersymmetric invariance depends on the order in which the lattice fields are written. Since the order of the lattice fields defines the symmetry transformation, this is just the statement that the invariance (or lack of it) depends on how one decides to transform the fields. I do not regard this as an inconsistency of the noncommutative approach, but rather as an illustration that in some theories there may be an ordering ambiguity that needs to be resolved in order to have the modified supersymmetric invariance. Indeed, Bruckmann and de Kok find that such an ordering does exist in the supersymmetric quantum mechanics model.

By contrast, the ordering in the D'Adda et al. approach is completely determined by the lattice gauge invariance. When the action is written in the form (2.4), there is no ambiguity resulting from the ordering of terms; $\Psi_{x, x}$, appearing in (2.4), is unique. The invariance of the component field action follows identically from the properties (2.4) and (2.5), and can made explicit using the $s_{A}$ transformations of fields given by D'Adda et al. All of this is easily checked by straightforward, if somewhat tedious, algebraic manipulations.

\subsubsection{Other no CT examples/claims}

Other recent examples where there is evidence presented that fine-tuning of CTs is avoided include: 
- Q-exact compact $(2,2)$ SYM in 2d as formulated by Sugino in [49-52]. There is no finetuning in perturbation theory.

- Twisted (Q-exact) geometrical (2,2) SYM in 2d, due to Catterall [53]. Monte Carlo data seems to indicate no need for CTs [54]. Of practical importance, the phase quenched ensemble in this model is a good approximation to the full ensemble, due to a sharply peaked phase distribution as one approaches the continuum limit.

- The 4d Wess-Zumino model with Ginsparg-Wilson fermions, recently proposed and studied by Bonini and Feo [55-57]. Here, there is a nonlinear, perturbative definition of the supercharges $Q_{A}$.

- The twisted (Q-exact) nonlinear 2d $\sigma$ model with $(2,2)$ supersymmetry [58]. A superfield formulation was subsequently given in [7], where renormalization was discussed on symmetry and dimensional grounds. Whereas that study showed that radiative generation of supersymmetry violation in the continuum limit is not forbidden by lattice symmetries and dimensional arguments, recent Monte Carlo data seems to indicate no need for supersymmetry-restoring CTs $[59,60]$.

In summary, much progress has been made recently in formulating supersymmetric theories on the lattice. In some cases, the theory has been nonperturbatively defined. Furthermore, numerical results have been obtained in a few cases, holding out the prospect that we will obtain interesting information by means other than the traditional, continuum methods.

\section{Nonholomorphic woes}

Now that we have seen that there are many promising formulations that define supersymmetric theories on the lattice, let us return to the motivations. Continuum supersymmetry tricks usually partly fail to determine the IR effective theory.

Schematically, the IR effective theory is described by an action of the form:

$$
\int d^{4} \theta K(\Phi, \bar{\Phi})+\left[\int d^{2} \theta W(\Phi)+\text { h.c. }\right]
$$

Here, integration over Grassmann superspace coordinates $\theta, \bar{\theta}$ has been used, and $\Phi, \bar{\Phi}$ are chiral superfields. The superpotential $W$ is not renormalized in perturbation theory. Also, $W$ is sometimes completely determined, once symmetries accounted for. Generically none of these nice features hold for the Kähler potential $K$.

This lack of control over nonholomorphic data, encoded in $K$, is distressing. This is because the so-called "supersymmetry-breaking soft-terms" largely determine superpartner spectra and couplings for the MSSM, and these soft-terms depend on the Kähler potential $K$. To the extent that nonperturbative physics is involved in breaking supersymmetry, the nonperturbative renormalization of the Kähler potential is important to the prediction of soft-terms.

Lattice Monte Carlo simulations would, as a first step, give us a handle on vevs $\phi_{0}$ of scalars, and the spectrum of light states. This constrains $\partial V / \partial \phi, \partial^{2} V / \partial \phi \partial \phi$ and $\partial^{2} V / \partial \phi \partial \bar{\phi}$ evalu- 
ated at $\phi_{0}$. Both the Kähler potential $K$ and superpotential $W$ play a role in the scalar potential, $V=K^{k \bar{\ell}} W_{k} \bar{W}_{\bar{\ell}}$, where $K^{k \bar{\ell}}$ is the inverse of the Kähler metric $K_{k \bar{\ell}}=\partial^{2} K / \partial \phi^{k} \partial \bar{\phi}^{\bar{\ell}}$, and $W_{k}=$ $\partial W / \partial \phi_{k}, \bar{W}_{\bar{\ell}}=\left(W_{\ell}\right)^{*}$.

A scheme for extracting the effective $K$ would be: (1) Hypothesize an effective Kähler potential $K$, containing some minimal set of phenomenological constants. (2) Use the known effective superpotential $W$. (3) Match microscopic lattice and IR effective lattice data, to fit "phenomenological" constants in $K$. This outline illustrates how lattice simulations have the potential to teach us something about nonperturbative renormalization of nonholomorphic quantities.

In work that is in progress with Simon Catterall, we are attempting to determine the $(2,2) 2 d$ twisted nonlinear $\sigma$ model that is the IR effective theory for $(2,2) 2 \mathrm{~d}$ SU(2) SYM. For example, we are comparing constrained effective potentials in the two theories, for various scalars operators.

\section{Supersymmetry breaking}

A third motivation is to improve our understanding of dynamical supersymmetry breaking. Strong supersymmetry dynamics is often invoked in models of soft supersymmetry breaking for the Minimal Supersymmetry Standard Model (MSSM). Any improvement of our understanding of the strong dynamics of supersymmetric gauge theories would be helpful.

As an example, consider $3 \mathrm{~d} \mathscr{N}=2 \mathrm{SYM}$. This is a model that is perhaps feasible for thorough lattice study. The theory has a simple content (one gluon, one adjoint Majorana fermion, one adjoint real scalar), so it will be efficient to simulate. It is a simple theory where the ground state supersymmetry is not yet fully understood. It is obtained from the $3 \mathrm{~d}$ reduction of $4 \mathrm{~d} \mathscr{N}=1 \mathrm{SYM}$.

This model was studied from the continuum in [61]. It was found that there is an instantongenerated potential for the modulus field $\phi$. However, there is uncertainty regarding the potential for small $\phi$, because the theory is strongly coupled in that regime. The only known supersymmetric vacuum is at $\phi \rightarrow \infty$. We would like to know: Is there a supersymmetric vacuum near origin in field space?

It seems promising to use a parity-preserving overlap-Dirac formulation, similar to $3 \mathrm{~d} \mathscr{N}=$ 1 model of [20]. If one does this, do we avoid fine-tuning, as in the $3 \mathrm{~d} \mathscr{N}=1$ case? It is a straightforward, open question to be explored.

\section{Quantum gravity and the AdS/CFT correspondence}

There is an evolving understanding of the relationship between SYM and string/M-theory. A nonperturbative formulation of string/M-theory in general backgrounds is still lacking. But there are recent successes for special backgrounds: D-branes [62]; M(atrix) theory [63,64]; the AdS/CFT correspondence [65-67]; and, the PP-wave limit [68-71]. Note that I include nontrivial semiclassical descriptions under what I call "nonperturbative." It is of considerable interest to study these nonpertubative formulations in relation to SYM on the lattice. I next survey an interesting example of the AdS/CFT correspondence. In this example, both the gauge and gravitational theories are known. Detailed information on the IR physics of the gauge theory, extracted from lattice studies, would nicely supplement the many continuum results that have been obtained. 


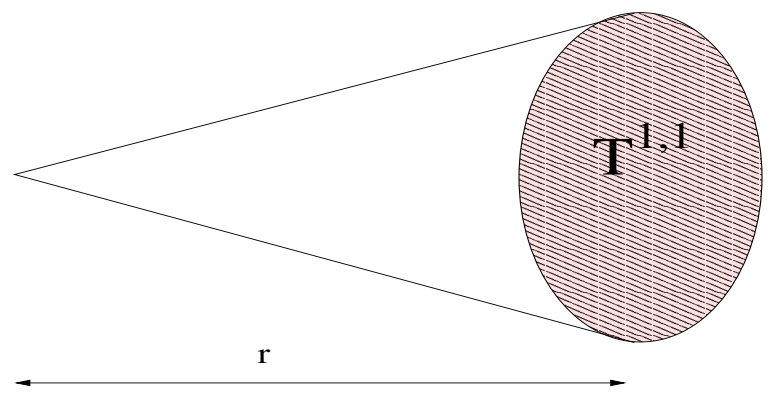

Figure 2: A schematic view of the conifold.
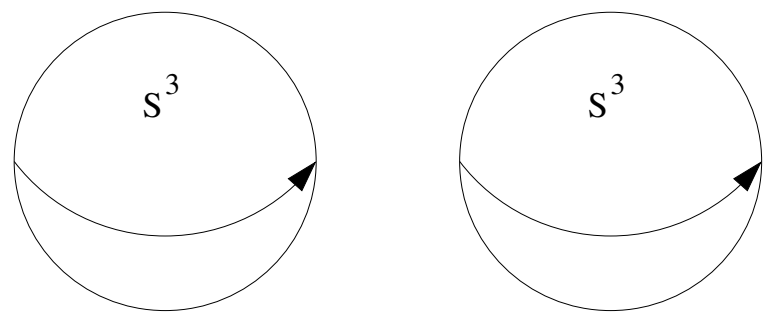

Figure 3: The quotient of $S^{3} \times S^{3}$ that leads to $T^{1,1}$.

AdS/CFT correspondence. Here, the nontrivial SYM vacuum dynamics takes on a gravitational meaning. Everybody has heard about $A d S_{5} \times S^{5}$. A very interesting generalization is the Klebanov-Strassler (KS) construction [72]. It is based on the Klebanov-Witten (KW) construction [73], with a gravitational theory on $A d S_{5} \times T^{1,1}$. The KW dual gauge theory is an $\mathscr{N}=1$ superconformal field theory (SCFT), whose field content and action is known.

The conifold. Type IIB string theory, and in particular its supergravity limit, is formulated on the 10d space:

$$
\text { 4d Minkowski } \times \text { conifold }
$$

The conifold is a $6 \mathrm{~d}$ space possessing a cone-like geometry, with $T^{1,1}$ base, as illustrated in Fig. 2 . The $5 \mathrm{~d}$ compact space $T^{1,1}$ is a quotient manifold:

$$
T^{1,1}=[S U(2) \times S U(2)] / U(1) .
$$

The " 1,1 " denotes the $U(1)$ quotient generated by:

$$
H=\left(\sigma_{3} \otimes \mathbf{1}\right)+\left(\mathbf{1} \otimes \sigma_{3}\right) .
$$

Fig. 3 illustrates the rotation, generated by $H$, that identifies the points in $S U(2) \times S U(2) \simeq S^{3} \times S^{3}$ to form $T^{1,1}$.

Warping spacetime. A stack of $N_{c}$ D3 branes is placed at the tip of the confold, where the size of the base shrinks to zero. I.e., $r^{2} d s_{T^{1,1}}^{2} \rightarrow 0$ as $r \rightarrow 0$. The D3 branes are gravitating, so they backreact on the geometry, warping it. As a consequence, not too far from the branes (the near-horizon limit), the geometry is $A d S_{5} \times T^{1,1}$. 

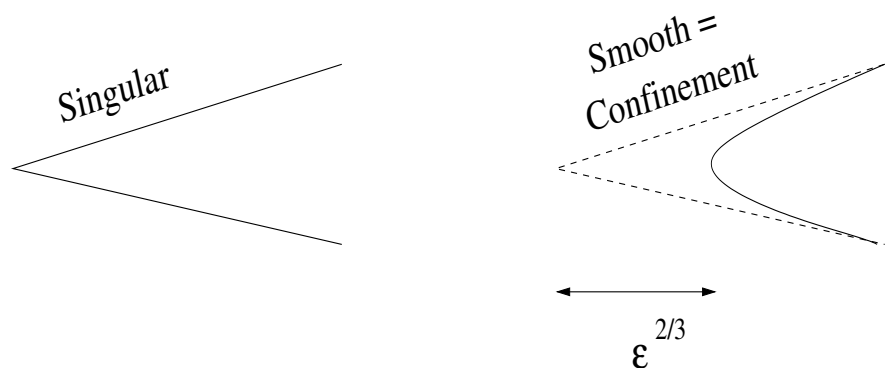

Figure 4: The singularity resolution in the deformed conifold. The parameter $\varepsilon$ is used to describe the geometric deformation, and makes an appearance in the glueball mass.

Killing spinors. One advantage of the conifold construction is that Type IIB SUGRA on $A d S_{5} \times T^{1,1}$ only preserves eight Killing spinors, whereas Type IIB on $A d S_{5} \times S^{5}$ preserves 32 Killing spinors. ${ }^{6}$ In the dual gauge theory, we get an $\mathscr{N}=1$ superconformal field theory rather than $\mathscr{N}=4$ superconformal field theory.

The singularity at the tip and its resolution. An $\mathscr{N}=1$ SCFT is a much more promising start, being closer to the real world. ${ }^{7}$ There is a singularity at the tip of the conifold, $r=0$. In the dual gauge theory, this is reflected by the absence of an IR cutoff. Klebanov-Strassler resolve the singularity with the deformed conifold, depicted schematically in Fig. 4. They show that this is equivalent to confinement in the IR of the dual gauge theory, due to running of gauge couplings once conformal symmetry (dual to the scaling isometry of AdS) is broken.

On the gravity side of the duality, the deformation of the conifold breaks half the Killing spinors. On the gauge theory side, this breaks the fermionic conformal charges, reducing to an $\mathscr{N}=1$ supersymmetric gauge theory that is not conformally invariant. Now we are "very close" to the real world; i.e., warped, supersymmetric extensions to the Standard Model. ${ }^{8}$

D7 probes and the equivalence to adding quark flavors. Introduction of $N_{f}$ D7 probe branes allows for a weakly coupled $U\left(N_{f}\right)$ gauge theory in the dual gauge theory, due to volume suppression associated with reduction to $4 \mathrm{~d}$ :

$$
g_{f}^{2} \sim(\text { volume })^{-1}
$$

The embedding for the D7 branes can generate bare masses for "quarks" of dual gauge theory. The low energy partons are really bound states, very much like in technicolor. ${ }^{9}$ Here, the $U\left(N_{c}\right)$ gauge theory associated with the D3 branes plays the role of the technicolor-like group.

Noncompact $\rightarrow$ compact Calabi-Yau. Recently, studies of the low energy effective $4 \mathrm{~d}$ and $5 \mathrm{~d}$ theories derived from the probe D7 branes have been conducted [75-78]. In the latter work [78], we also imagined a regulator in the UV, following [79]. This occurs by capping off the conifold with a compact Calabi-Yau manifold far from the tip, as in Fig. 5.

\footnotetext{
${ }^{6}$ The number of Killing spinors determines the amount of supersymmetry in the supergravity theory.

${ }^{7} \mathscr{N}=1$ is necessary in order to have chiral representations.

${ }^{8}$ For a review of phenomenological models of this type, see [74].

${ }^{9}$ Unlike technicolor models, masses for the quarks associated with probe D7 branes are allowed, because the spec-
} 


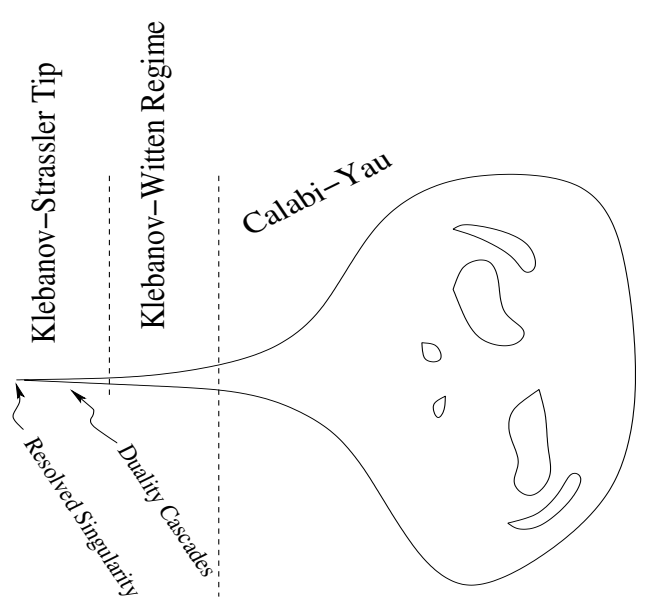

Figure 5: A combination of a Klebanov-Strassler throat and a Calabi-Yau "boundary". This can be thought of as a refinement of Randall-Sundrum 1 type models.

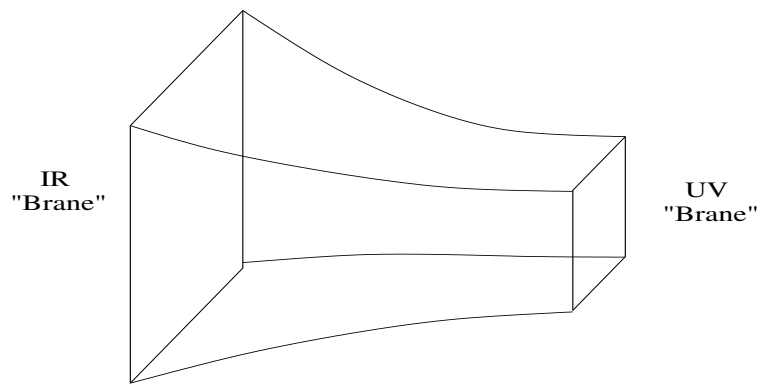

Figure 6: A schematic picture of the background geometry in the Randall-Sundrum 1 model [80]. The figure is meant to indicate that, at the IR brane, distances are dilated by the warp factor of AdS.

Randall-Sundrum 1 interpretation. The Calabi-Yau and the KS tip are, respectively, to be thought of as refinements of the UV and IR branes of, say, Randall-Sundrum 1 type models, shown schmatically in Fig. 6. The refined picture, which has already been mentioned above, is shown in Fig. 5. There is a potential—though challenging-interplay between warped extra dimension models, AdS/CFT, and lattice SYM. A detailed study of the IR physics of the dual gauge theory would have implications for the gravitational picture. Thus, the goal is to be able to simulate the gauge theory on the lattice.

\section{6. $A d S_{3} / C F T_{2}$ and deconstructed $(4,4)$ super-QCD}

There are many more generalizations of AdS/CFT. In particular, $A d S_{3} / C F T_{2}$ may be accessible through lattice studies. The duality in the $2 \mathrm{~d}(4,4)$-supersymmetric QCD (the D1-D5 brane intersection theory) is currently under study using a deconstruction lattice supersymmetry

trum is vector-like. 
approach [41]. The $(4,4)$ lattice with matter is a generalization of the recent $(2,2)$ super-QCD constructions of Kaplan (see talk, this proceedings) and Endres [40]. The $(4,4)$ lattice theory has two exact supercharges and only site, ordinary link, and diagonal link fields.

\section{Conclusions}

Although it is challenging to write down supersymmetric lattice field theories that have a "good" quantum continuum limit, some examples do exist. A wealth of exciting applications await. The most interesting are those related to supersymmetry breaking, quantum gravity and the AdS/CFT correspondence. Certain nonpertubative features, such as spectra, can be studied on the lattice with results that are of broad and current interest. For practical reasons, and due to the existence of very promising formulations, the $2 \mathrm{~d}$ lattice models could be the most fruitful numerically, in the short term.

\section{Acknowledgements}

Thanks are due to Erich Poppitz, David B. Kaplan, Herbert Neuberger, Pavlos Vranas, Alessandro D'Adda, Noboru Kawamota, Simon Catterall, Falk Bruckmann and Mark de Kok for helpful communications. This work was supported in part by the U.S. Department of Energy under grant No. DE-FG02-94ER-40823.

\section{References}

[1] J. Giedt, Int. J. Mod. Phys. A 21 (2006) 3039 [arXiv:hep-lat/0602007].

[2] S. Catterall, PoS LAT2005 (2006) 006 [arXiv:hep-lat/0509136].

[3] A. Feo, Mod. Phys. Lett. A 19 (2004) 2387 [arXiv:hep-lat/0410012].

[4] A. Feo, Nucl. Phys. Proc. Suppl. 119 (2003) 198 [arXiv:hep-lat/0210015].

[5] I. Montvay, Int. J. Mod. Phys. A 17 (2002) 2377 [arXiv:hep-lat/0112007].

[6] S. Catterall and E. Gregory, Phys. Lett. B 487 (2000) 349 [arXiv:hep-lat/0006013].

[7] J. Giedt and E. Poppitz, JHEP 0409, 029 (2004) [arXiv:hep-th/0407135].

[8] J. Wess and B. Zumino, Phys. Lett. B 49 (1974) 52.

[9] S. Elitzur and A. Schwimmer, Nucl. Phys. B 226, 109 (1983).

[10] N. Sakai and M. Sakamoto, Nucl. Phys. B 229, 173 (1983).

[11] T. Reisz, Commun. Math. Phys. 116 (1988) 81.

[12] M. Beccaria, G. Curci and E. D’Ambrosio, Phys. Rev. D 58 (1998) 065009 [arXiv:hep-lat/9804010].

[13] S. Catterall and S. Karamov, Phys. Rev. D 65 (2002) 094501 [arXiv:hep-lat/0108024].

[14] S. Catterall and S. Karamov, Nucl. Phys. Proc. Suppl. 106 (2002) 935 [arXiv:hep-lat/0110071].

[15] J. Giedt, Nucl. Phys. B 726 (2005) 210 [arXiv:hep-lat/0507016].

[16] G. Curci and G. Veneziano, Nucl. Phys. B 292 (1987) 555. 
[17] R. Narayanan and H. Neuberger, Phys. Lett. B 302 (1993) 62 [arXiv:hep-lat/9212019].

[18] R. Narayanan and H. Neuberger, Nucl. Phys. B 412 (1994) 574 [arXiv:hep-lat/9307006].

[19] H. Neuberger, Phys. Lett. B 417 (1998) 141 [arXiv:hep-lat/9707022].

[20] N. Maru and J. Nishimura, Int. J. Mod. Phys. A 13 (1998) 2841 [arXiv:hep-th/9705152].

[21] H. Neuberger, Phys. Rev. D 57 (1998) 5417 [arXiv:hep-lat/9710089].

[22] D. B. Kaplan, Phys. Lett. B 288 (1992) 342 [arXiv:hep-lat/9206013].

[23] J. Nishimura, Phys. Lett. B 406 (1997) 215 [arXiv:hep-lat/9701013].

[24] D. B. Kaplan and M. Schmaltz, Chin. J. Phys. 38 (2000) 543 [arXiv:hep-lat/0002030].

[25] G. T. Fleming, J. B. Kogut and P. M. Vranas, Phys. Rev. D 64 (2001) 034510 [arXiv:hep-lat/0008009].

[26] G. Veneziano and S. Yankielowicz, Phys. Lett. B 113 (1982) 231.

[27] G. R. Farrar, G. Gabadadze and M. Schwetz, Phys. Rev. D 58 (1998) 015009 [arXiv:hep-th/9711166].

[28] G. R. Farrar, G. Gabadadze and M. Schwetz, Phys. Rev. D 60 (1999) 035002 [arXiv:hep-th/9806204].

[29] G. Gabadadze, Nucl. Phys. B 544 (1999) 650 [arXiv:hep-th/9808005].

[30] D. G. Cerdeno, A. Knauf and J. Louis, Eur. Phys. J. C 31 (2003) 415 [arXiv:hep-th/0307198].

[31] P. Merlatti and F. Sannino, Phys. Rev. D 70 (2004) 065022 [arXiv:hep-th/0404251].

[32] R. Auzzi and F. Sannino, Phys. Rev. D 72 (2005) 105003 [arXiv:hep-th/0509166].

[33] P. M. Vranas, Phys. Rev. D 74 (2006) 034512 [arXiv:hep-lat/0606014]; see also talk in this proceedings.

[34] N. Arkani-Hamed, A. G. Cohen and H. Georgi, Phys. Rev. Lett. 86, 4757 (2001) [arXiv:hep-th/0104005];

[35] C. T. Hill, S. Pokorski and J. Wang, Phys. Rev. D 64, 105005 (2001) [arXiv:hep-th/0104035].

[36] D. B. Kaplan, E. Katz and M. Unsal, JHEP 0305 (2003) 037 [arXiv:hep-lat/0206019].

[37] A. G. Cohen, D. B. Kaplan, E. Katz and M. Unsal, JHEP 0308, 024 (2003) [arXiv:hep-lat/0302017].

[38] A. G. Cohen, D. B. Kaplan, E. Katz and M. Unsal, JHEP 0312, 031 (2003) [arXiv:hep-lat/0307012].

[39] D. B. Kaplan and M. Unsal, JHEP 0509 (2005) 042 [arXiv:hep-lat/0503039].

[40] M. G. Endres and D. B. Kaplan, arXiv:hep-lat/0604012.

[41] J. Giedt, arXiv:hep-lat/0605004.

[42] T. Onogi and T. Takimi, Phys. Rev. D 72 (2005) 074504 [arXiv:hep-lat/0506014].

[43] J. Giedt, Nucl. Phys. B 668 (2003) 138 [arXiv:hep-lat/0304006].

[44] J. Giedt, Nucl. Phys. B 674 (2003) 259 [arXiv:hep-lat/0307024].

[45] J. Giedt, arXiv:hep-lat/0405021.

[46] A. D’Adda, I. Kanamori, N. Kawamoto and K. Nagata, Nucl. Phys. B 707 (2005) 100 [arXiv:hep-lat/0406029].

[47] A. D’Adda, I. Kanamori, N. Kawamoto and K. Nagata, Phys. Lett. B 633 (2006) 645 [arXiv:hep-lat/0507029]. 
[48] F. Bruckmann and M. de Kok, Phys. Rev. D 73 (2006) 074511 [arXiv:hep-lat/0603003].

[49] F. Sugino, JHEP 0401 (2004) 015 [arXiv:hep-lat/0311021].

[50] F. Sugino, JHEP 0403 (2004) 067 [arXiv:hep-lat/0401017].

[51] F. Sugino, JHEP 0501 (2005) 016 [arXiv:hep-lat/0410035].

[52] F. Sugino, Phys. Lett. B 635 (2006) 218 [arXiv:hep-lat/0601024].

[53] S. Catterall, JHEP 0411 (2004) 006 [arXiv:hep-lat/0410052].

[54] S. Catterall, JHEP 0603 (2006) 032 [arXiv:hep-lat/0602004].

[55] M. Bonini and A. Feo, JHEP 0409 (2004) 011 [arXiv:hep-lat/0402034].

[56] M. Bonini and A. Feo, Nucl. Phys. Proc. Suppl. 140, 766 (2005) [arXiv:hep-lat/0409068].

[57] M. Bonini and A. Feo, Phys. Rev. D 71 (2005) 114512 [arXiv:hep-lat/0504010].

[58] S. Catterall and S. Ghadab, JHEP 0405 (2004) 044 [arXiv:hep-lat/0311042].

[59] S. Ghadab, Nucl. Phys. Proc. Suppl. 140 (2005) 760 [arXiv:hep-lat/0409102].

[60] S. Catterall and S. Ghadab, arXiv:hep-lat/0607010.

[61] I. Affleck, J. A. Harvey and E. Witten, Nucl. Phys. B 206 (1982) 413.

[62] J. Polchinski, Phys. Rev. Lett. 75 (1995) 4724 [arXiv:hep-th/9510017].

[63] T. Banks, W. Fischler, S. H. Shenker and L. Susskind, Phys. Rev. D 55 (1997) 5112 [arXiv:hep-th/9610043].

[64] N. Ishibashi, H. Kawai, Y. Kitazawa and A. Tsuchiya, Nucl. Phys. B 498 (1997) 467 [arXiv:hep-th/9612115].

[65] J. M. Maldacena, Adv. Theor. Math. Phys. 2 (1998) 231 [Int. J. Theor. Phys. 38 (1999) 1113] [arXiv:hep-th/9711200].

[66] S. S. Gubser, I. R. Klebanov and A. M. Polyakov, Phys. Lett. B 428 (1998) 105 [arXiv:hep-th/9802109].

[67] E. Witten, Adv. Theor. Math. Phys. 2 (1998) 253 [arXiv:hep-th/9802150].

[68] R. R. Metsaev, Nucl. Phys. B 625 (2002) 70 [arXiv:hep-th/0112044].

[69] R. R. Metsaev and A. A. Tseytlin, Phys. Rev. D 65 (2002) 126004 [arXiv:hep-th/0202109].

[70] M. Blau, J. Figueroa-O'Farrill, C. Hull and G. Papadopoulos, JHEP 0201 (2002) 047 [arXiv:hep-th/0110242].

[71] D. Berenstein, J. M. Maldacena and H. Nastase, JHEP 0204 (2002) 013 [arXiv:hep-th/0202021].

[72] I. R. Klebanov and M. J. Strassler, JHEP 0008 (2000) 052 [arXiv:hep-th/0007191].

[73] I. R. Klebanov and E. Witten, Nucl. Phys. B 536 (1998) 199 [arXiv:hep-th/9807080].

[74] For a review, see T. Gherghetta, arXiv:hep-ph/0601213.

[75] T. Sakai and J. Sonnenschein, JHEP 0309 (2003) 047 [arXiv:hep-th/0305049].

[76] S. Kuperstein, JHEP 0503 (2005) 014 [arXiv:hep-th/0411097].

[77] T. S. Levi and P. Ouyang, arXiv:hep-th/0506021.

[78] T. Gherghetta and J. Giedt, Phys. Rev. D74 (2006) 066007 [hep-th/0605212].

[79] S. B. Giddings, S. Kachru and J. Polchinski, Phys. Rev. D 66 (2002) 106006 [arXiv:hep-th/0105097].

[80] L. Randall and R. Sundrum, Phys. Rev. Lett. 83 (1999) 3370 [arXiv:hep-ph/9905221]. 